\title{
FIXED POINT THEOREMS AND VARIATIONAL INEQUALITIES WITH NON-COMPACT SETS
}

\author{
FANG-NI XIANG \\ Department of Basic Courses \\ Gezhouba Institute of Hydro-electric Engineering \\ Yichang, Hubei 443002, P R China \\ and
}

LOKENATH DEBNATH

Department of Mathematics

University of Central Florida

Orlando, FL 32816-1364, U S A

(Received July 13,1993 and in revised form February 21, 1994)

\begin{abstract}
This paper deals with a generalization of Browder fixed point theorems and variational inequalities by relaxing the assumptions of compactness and continuity
\end{abstract}

KEY WORDS AND PHRASES. Fixed points, variational inequalities, and non-compact set 1991 AMS SUBJECT CLASSIFICATION CODE(S). 49A29, 49A10.

\section{INTRODUCTION.}

Browder [1] proved the following remarkable fixed point theorem

THEOREM 1.1 Let $K$ be a compact convex subset of a real Hausdorff topological vector space $E$ Let $T$ be a mapping of $K$ into $2^{K}$, where for each $x \in K, T(x)$ is a non-empty convex subset of $K$ Suppose further that for each $y$ in $K, T^{-1}(y)=\{x: x \in K, y \in T(x)\}$ is open in $K$. Then there exists $x^{*}$ in $K$, such that $x^{*}$ is in $T\left(x^{*}\right)$.

Browder applied his theorem to obtain the following variational inequality.

THEOREM 1.2. Let $K$ be a non-empty compact convex subset of the locally convex Hausdorff topological vector space $E, T$ an upper semicontinuous mapping of $K$ into $2^{E^{*}}$ such that for each $x$ in $K, T(x)$ is a non-empty compact convex subset of $E^{*}$. Then there exists a $u_{0}$ in $K$ and $w_{0} \in T(u)$ such that

$$
\left(w_{0}, u_{0}-u\right) \geq 0, \quad \text { for all } u \text { in } K .
$$

Subsequently, Browder's theorem has been generalized by a number of authors including Tian [2], Tarafdar ([3], [4] and [5]) and Lassonde [6]

The main purpose of this paper is to extend these results of Browder by relaxing the assumptions of compactness and continuity. Our main results are Theorems 3.1 and 4.1. It is shown that our Theorems 32 and 33 are direct consequences of Theorem 31 Finally, it is pointed out that our results are different from the corresponding results of Tarafdar, and Lassonde. 


\section{DEFINITIONS.}

Let $X$ and $Y$ be two topological vector spaces, and let $2^{Y}$ be the set of all subset of $Y$

A correspondence $G: X \rightarrow 2^{Y}$ is said to be upper semicontinuous (in short, u s c) if for each $x$ in $D o m G$ and any neighborhood $V$ of $G(x)$, there exists a neighborhood $U$ of $x$ such that

$$
G(u) \subset V, \text { for all } u \text { in } U
$$

We say that $G: X \rightarrow 2^{Y}$ is upper hemicontımuous (in short, $\mathrm{u} \mathrm{h} \mathrm{c} \mathrm{)} \mathrm{if} \mathrm{for} \mathrm{each} x$ in DomG and for any $p$ in $Y^{*}$ (dual of $Y$ ), the functional

$$
x \rightarrow \sigma(G(x), p)=\sup _{y \in G(x)}(p, y)
$$

is upper semicontinuous, where $\sigma$ is called the upper support function of $G$ and $D o m G=\{x \in X: G(x) \neq \emptyset\}$.

We recall that a real-valued function $f$ defined on a convex set $C$ in $X$ is said to be quasiconvex if, for every real number $t$, the set $\{x \in C: f(x)<t\}$ is convex

REMARK 1. It will be noted that for a correspondence $G: X \rightarrow 2^{Y}$ with non-empty closed convex values, the u s c implies the $\mathrm{u} \mathrm{h} \mathrm{c.,} \mathrm{but} \mathrm{the} \mathrm{converse} \mathrm{is} \mathrm{not} \mathrm{necessarily} \mathrm{true} \mathrm{(see} \mathrm{also} \mathrm{Jian} \mathrm{[7])}$

\section{FIXED POINT THEOREMS.}

THEOREM 3.1. Let $E$ be a Hausdorff topological vector space ( $E$ not necessarily locally convex), $X$ is a non-empty subset of $E, F: X \rightarrow 2^{E}$, where for each $x$ in $X, F(x)$ is a non-empty convex subset of $E$ and for every $y$ in $E, F^{-1}(y)=\{x: x \in X, y \in F(x)\}$ is open in $X$ Then the necessary and sufficient condition for the existence of fixed point $x^{*} \in F\left(x^{*}\right)$ is that there exists a non-empty compact convex subset $K \subset X$ such that

$$
F(x) \cap K \neq \emptyset \quad \text { for all } x \text { in } K
$$

PROOF. The condition is necessary Suppose $F$ has a fixed point $x^{*} \in F\left(x^{*}\right)$ and let $K=\left\{x^{*}\right\}$ Then the subset $K$ is clearly compact and convex and $F(x) \cap K$ is non-empty for all $x$ in $K$

The condition is sufficient Let the mapping $T: K \rightarrow 2^{K}$ and

$$
T(x)=F(x) \cap K, \quad \text { for all } x \text { in } K
$$

Then for every $y$ in $K, T^{-1}(y)$ is open in $K$ by lemma 2 proved by Tian [2] Obviously, $T(x)$ is nonempty convex subset of $K$ Using Theorem 11 , we can say that there exists a fixed point $x^{*}$ in $K$ such that is $x^{*}$ is in $T\left(x^{*}\right)=F\left(x^{*}\right) \cap K$. So there exists a point $x^{*} \in X$, such that $x^{*} \in F\left(x^{*}\right)$.

As a direct corollary of Theorem 3.1, the following result of Tian ([2], theorem 2) follows as.

THEOREM 3.2. Let $X$ be non-empty paracompact subset in a locally convex Hausdorff topological vector space $E$. Suppose that $F: X \rightarrow 2^{E}$, for every $x$ in $X, F(x)$ is non-empty closed convex subset of $E$, further, for any $y$ in $E, F^{-1}(y)=\{x \in X: y \in F(x)\}$ is open in $X$. Then the necessary and sufficient condition for the existence of a fixed point $x^{*} \in F\left(x^{*}\right)$ is that there exists a nonempty compact convex subset $K \subseteq X$ such that

$$
F(x) \cap K \neq \emptyset, \quad \text { for all } x \text { in } K .
$$

We next use Theorem 3.1 to prove the following theorem which is different from $\mathrm{Ky}$ ([8], Lemma 1) and Takahashi ([9], Theorem 15)

THEOREM 3.3. Let $E$ be a Hausdorff topological vector space and $X$ is a non-empty subset of $\mathrm{E}$ Let $A$ be a subset of $X \times X$ having the following properties:

(1) For any $y \in X$, the set $\{x \in X:(x, y) \in A\}$ is closed;

(2) for any $x \in X$, the set $\{y \in X:(x, y) \notin A\}$ is convex (or empty);

(3) $(x, x) \in A$, for every $x$ in $X$;

(4) For a non-empty compact convex subset $K$ in $X$ with each $x \in X$, there exists $y \in K$ and $(x, y) \notin A$. 
Then, there exists a $x^{*}$ in $X$, such that $\left\{x^{*}\right\} \times X \subset A$

PROOF. For each $x$ in $X$, we set the mapping $S . X \rightarrow 2^{X}$ defined by

$$
S(x)=\{y \in X:(x, y) \notin A\}
$$

There exists a $x^{*}$ in $X$ with $S\left(x^{*}\right)=\emptyset$ from the definition of the mapping $S$ We have $\left(x^{*}, y\right) \in A$, for all $y$ in $X$, that is $\left\{x^{*}\right\} \times X \subset A$. If $S(x) \neq \emptyset$ for all $x$ in $X$, then $S(x)$ is convex

And $S^{1}(y)=\{x \in X: y \in S(x)\}=X \backslash\{x \in X .(x, y) \in A\}$ is open in $X$ For compact convex subset $K$, we have $S(x) \cap K \neq \emptyset$ for all $x$ in $K$ by the assumed conditions Then by Theorem 31 , there exists $a \in S(a)$, that is $(a, a) \notin A$, which is a contradiction Therefore, we have $S\left(x^{*}\right)=\emptyset$ for some $x^{*}$ in $X$, so there exists a $x^{*}$ in $X$, such that $\left\{x^{*}\right\} \times X \subset A$. This completes the proof

\section{VARIATIONAL INEQUALITIES.}

In this section, we generalize the variational inequality $\left(\begin{array}{ll}1 & 1\end{array}\right)$ as stated in Theorem 12 by relaxing the compactness and continuity conditions To prove our result, we need a lemma which was originally established by Allen [10]

ALLEN'S LEMMA 4.1 Let $E$ be a topological vector space, let $X$ be a non-empty convex subset of $E$, and let $f$ be a real-valued function on $X \times X$ Suppose that

(a) $f(x, x) \geq 0$, for all $x$ in $X$,

(b) for each fixed $x$ in $X$, the mapping $y \rightarrow f(x, y)$ is quasiconvex on $X$,

(c) for each fixed $y$ in $X$, the mapping $x \rightarrow f(x, y)$ is upper semicontinuous on $X$,

(d) there exists a non-empty compact convex set $K$ in $X$ such that for each $x$ in $X \backslash K$, there exists $y$ in $K$ with $f(x, y)<0$.

Then, there exists a $x^{*}$ in $K$ such that

$$
f\left(x^{*}, y\right) \geq 0, \quad \text { for all } y \text { in } X .
$$

By using this Lemma, we prove the following variational inequality which generalize the Theorem 12

THEOREM 4.1 Let $X$ be a non-empty convex subset of the locally convex Hausdorff topological vector space $E, T$ an upper hemicontinuous mapping of $X$ into $2^{E^{*}}, T(x)$ is a non-empty compact convex subset of $E^{*}$. Further, there exists a non-empty compact convex subset $K \subset X$, such that for each $x$ in $X \backslash K$, there exists a $y$ in $K$ with sup $\quad(w, x-y)<0 \quad$ Then there exists a $x^{*}$ in $X$ and a $w^{*}$ in $T\left(x^{*}\right)$ such that

$$
w \in T(x)
$$

$$
\left(w^{*}, x^{*}-y\right) \geq 0 \quad \text { for all } y \text { in } X
$$

PROOF. Define a real valued function $f$ on $X \times X$ by

$$
f(x, y)=\sup _{w \in T(x)}(w, x-y)
$$

Then, by upper hemicontinuity of $T$, we have that mapping $x \rightarrow f(x, y)$ is upper semicontinuous (see Tian and Zhou [11], Theorem 7) It is obvious that $y \rightarrow f(x, y)$ is convex and $f(x, x)=0$ for all $x$ in $X$ Further, $f(x, y)$ satisfies the condition (d) of Allen's Lemma 4.1 on compact convex subset $K$ Hence, by Allen's Lemma, there exists a $x^{*}$ in $X$ such that

$$
\sup _{w \in T\left(x^{*}\right)}\left(w, x^{*}-y\right) \geq 0 \quad \text { for all } y \text { in } X
$$

Since $T\left(x^{*}\right)$ is compact convex subset, there exists a $w^{*}$ in $T\left(x^{*}\right)$ such that $\left(w^{*}, x^{*}-y\right) \geq 0$ for all $y$ in $X$

REMARK 2. The "coercivity" assumption of Theorem 41 can be replaced by the weaker condition as

There exists a non-empty compact convex subset $K \subset X$, such that for each $x$ in $X \backslash K$ and each $w \in T(x)$, there exists a $y$ in $K$ with $(w, x-y)<0$. 
In fact, by applying the Lemma 1 of Browder [1] and the proof of Theorem 41 from the above assumption, we can obtain the "coercivity" condition of Theorem 41 So we have the next theorem

THEOREM 4.2. Let $K, X, E$ and $T$ are the same as in Theorem 41 Furthermore, suppose that for each $x$ in $X \backslash K$ and each $w \in T(x)$, there exists a $y$ in $K$, such that $(w, x-y)<0$.

Then there exists a $x^{*}$ in $X$ and $w^{*} \in T\left(x^{*}\right)$, such that

$$
\left(w^{*}, x^{*}-y\right) \geq 0, \quad \text { for all } y \text { in } X \text {. }
$$

REMARK 3. In case of a single-valued mapping, Theorem 42 take the following form

COROLlARY 1 (Allen [10]). Let $E$ be a topological vector space, let $X \subset E$ be a convex subset Let $T: X \rightarrow E^{*}$ be a mapping such that the mapping $x \rightarrow(T x, x)$ is upper semicontinuous on $X$ Let $K \subset X$ be a non-empty compact convex subset and suppose that for $x \in X \backslash K$ there exists $y$ in $K$ such that $(T x, x-y)<0$. Then there exists a vector $x^{*}$ in $K$ such that

$$
\left(T x^{*}, x^{*}-y\right) \geq 0, \quad \text { for all } y \text { in } X \text {. }
$$

\section{REFERENCES}

1 BROWDER, FE, The fixed point theory of multi-valued mapping in topological vector space, Math. Annal. 177 (1968), 283-301

2. TIAN, G, Fixed point theorem for mapping with non-compact and non-convex domains, J. Math. Anal. Appl. 158 (1991), 161-167

3 TARAFDAR, E, Five equivalent theorems on a convex subset of a topological vector space, Comment. Math. Univ. Carolinae 30 (1989), 323-326

4 TARAFDAR, E, On nonlinear variational inequalities, Proc. Amer. Math. Soc. 67 (1977), 95-98

5 TARAFDAR, E, A fixed point theorem equivalent to Fan-KKM theorem, J. Math. Anal. Appl. 128 (1987), 475-497

6 LASSONDE, M, On the use of KKM multifunctions in fixed point theory and related topic, $J$. Math. Anal. Appl. 97 (1983), 151-201

7 JIAN, Y, Two examples on continuity of multi-valued mapping, J. Math. Research and Exposition 1 (1990), 44-46

$8 \mathrm{KY}, \mathrm{F}$, Extension of two fixed point theorems of F E Browder, Math. Z. 112 (1969), 234-240

9 TAKAHASHI, W., Nonlinear variational inequalities and fixed point theorems, J. Math. Soc. Japan. 28 (1976), 168-181

10 ALLEN, G, Variational inequalities complementarity problems and duality theorems, J. Math. Anal. Appl. $\mathbf{5 8}$ (1977), 1-10

11. TIAN, G., and ZHOU, X., Quasi-variational inequalities with non-compact sets, J. Math. Anal. Appl. 160 (1991), 583-595 


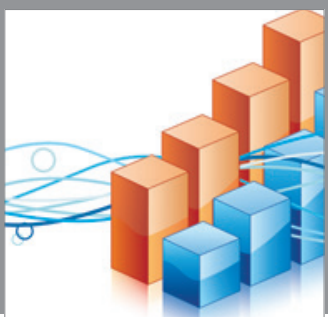

Advances in

Operations Research

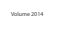

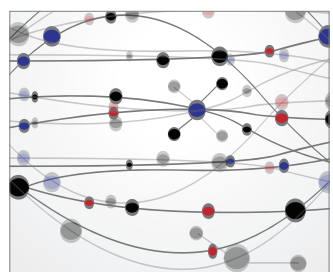

\section{The Scientific} World Journal
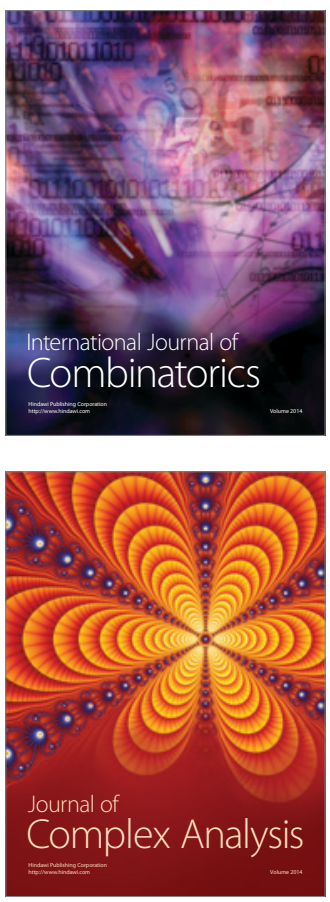

International Journal of

Mathematics and

Mathematical

Sciences
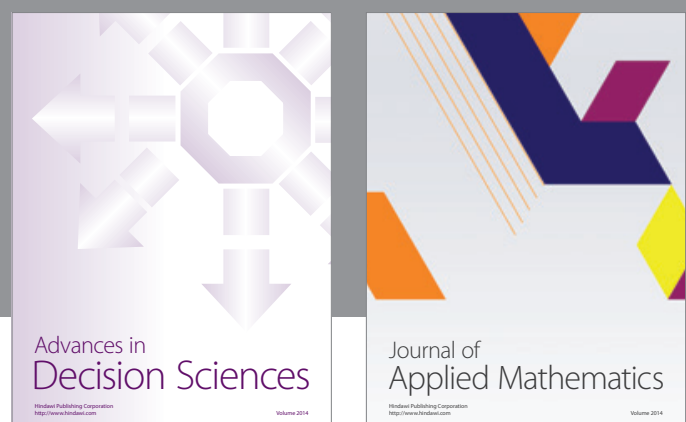

Journal of

Applied Mathematics
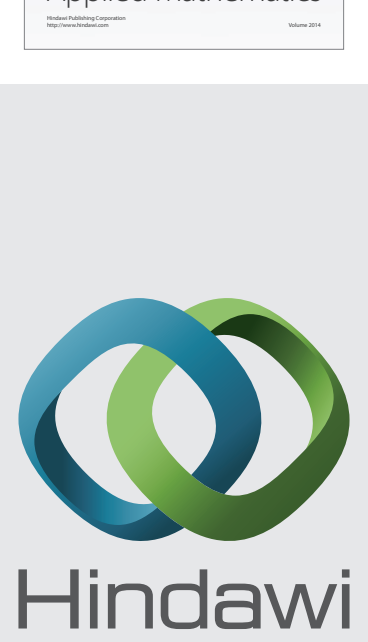

Submit your manuscripts at http://www.hindawi.com
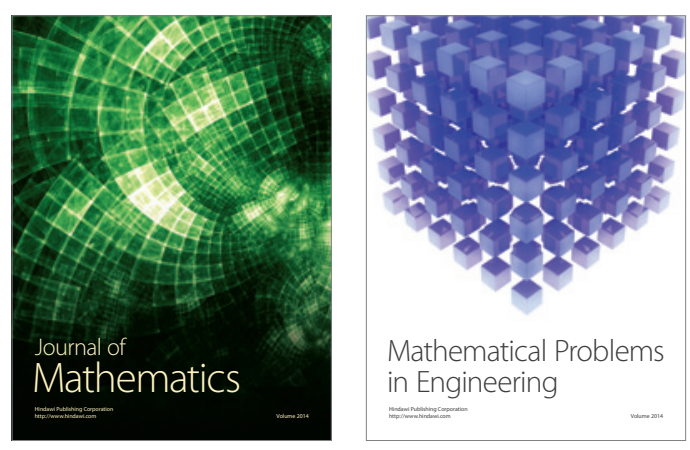

Mathematical Problems in Engineering
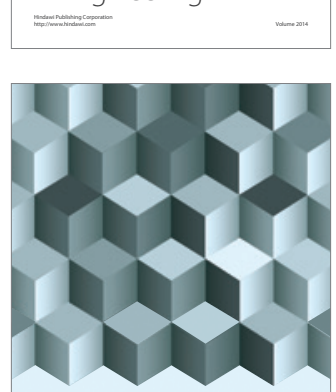

Journal of

Function Spaces
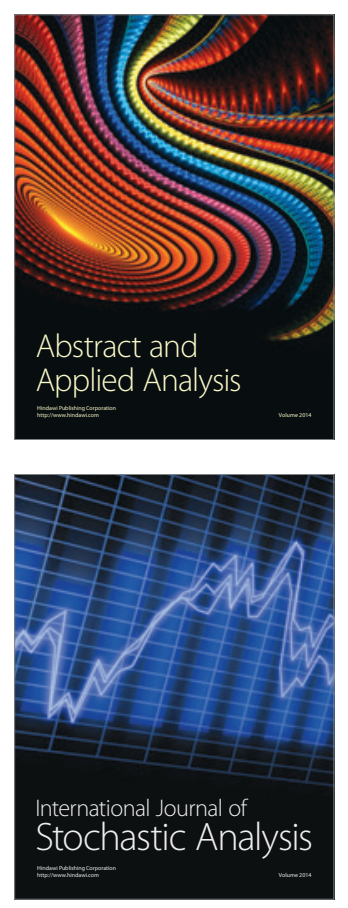

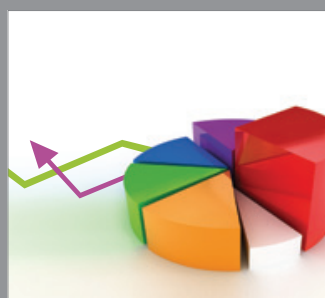

ournal of

Probability and Statistics

Promensencen
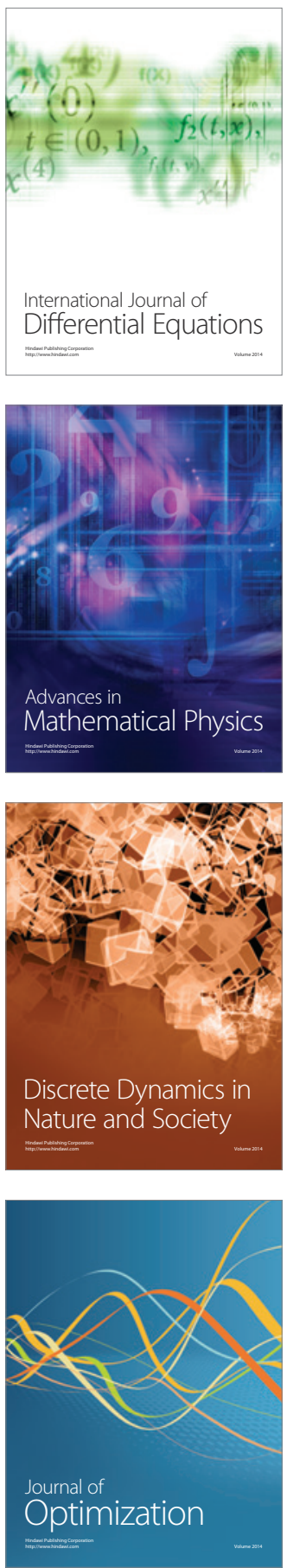